\title{
Rank-Crank type PDE's for higher level Appell functions
}

by

SANDER Zwegers (Dublin)

1. Introduction and statement of results. Dyson in [5] introduced the rank of a partition, to explain the first two of the three Ramanujan congruences

$$
\begin{aligned}
p(5 n+4) & \equiv 0(\bmod 5), \\
p(7 n+5) & \equiv 0(\bmod 7), \\
p(11 n+6) & \equiv 0(\bmod 11) .
\end{aligned}
$$

Here $p(n)$ denotes the number of partitions of $n$. He defined the rank of a partition as the largest part minus the number of its parts and conjectured that the partitions of $5 n+4$ (resp. $7 n+5)$ form 5 (resp. 7 ) groups of equal size when sorted by their ranks modulo 5 (resp. 7 ). This was later proven by Atkin and Swinnerton-Dyer in [3]. We are interested here in the generating function

$$
R(w ; q):=\sum_{\lambda} w^{\operatorname{rank}(\lambda)} q^{\|\lambda\|}=\frac{1-w}{(q)_{\infty}} \sum_{n \in \mathbb{Z}} \frac{(-1)^{n} q^{\frac{n}{2}(3 n+1)}}{1-w q^{n}},
$$

where $(q)_{\infty}:=\prod_{n=1}^{\infty}\left(1-q^{n}\right)$. In the first sum the $\lambda$ run over all partitions, $\operatorname{rank}(\lambda)$ denotes the rank of $\lambda$ and $\|\lambda\|$ denotes the size of the partition (the sum of all its parts).

Another partition statistic is the so called crank of a partition. For the generating function we have

$$
\begin{aligned}
C(w ; q) & :=\sum_{\lambda} w^{\operatorname{crank}(\lambda)} q^{\|\lambda\|}=\prod_{n=1}^{\infty} \frac{1-q^{n}}{\left(1-w q^{n}\right)\left(1-w^{-1} q^{n}\right)} \\
& =\frac{1-w}{(q)_{\infty}} \sum_{n \in \mathbb{Z}} \frac{(-1)^{n} q^{\frac{n}{2}(n+1)}}{1-w q^{n}}=(1-w) \frac{(q)_{\infty}^{2}}{\sum_{n \in \mathbb{Z}}(-1)^{n} q^{\frac{n}{2}(n-1)} w^{n}} .
\end{aligned}
$$

2010 Mathematics Subject Classification: 11F11, 11F27, 11F50.

Key words and phrases: modular forms, (mock) Jacobi forms, Appell functions. 
The crank was introduced by Andrews and Garvan in [1 to explain the Ramanujan congruence (1.1) with modulus 11.

In the setting of Jacobi forms it is more natural to consider the following modified rank and crank generating functions

$$
\mathcal{R}(z ; \tau):=\frac{w^{1 / 2} q^{-1 / 24}}{1-w} R(w ; q), \quad \mathcal{C}(z ; \tau):=\frac{w^{1 / 2} q^{-1 / 24}}{1-w} C(w ; q) .
$$

Here we use $w=\exp (2 \pi i z)$ and $q=\exp (2 \pi i \tau)$, with $z \in \mathbb{C}$ and $\tau$ in the complex upper half plane $\mathbb{H}$.

REMARK 1.1. $\mathcal{C}$ is a meromorphic Jacobi form of weight $1 / 2$ and index $-1 / 2$ and in [10] it is shown that $\mathcal{R}$ is a mock Jacobi form of weight $1 / 2$ and index $-3 / 2$.

For the theory of Jacobi forms we refer the reader to [6]. Although, some of the formulas and methods presented here are motivated by Jacobi forms, we will actually not use many of their properties. For the purpose of this paper it is also not necessary to know what a mock Jacobi form is.

The two modified generating functions are related by a partial differential equation, which we will refer to as the Rank-Crank PDE.

Theorem 1.2 (see [2]). If we define the heat operator $\mathrm{H}$ by

$$
\mathrm{H}:=\frac{3}{\pi i} \frac{\partial}{\partial \tau}+\frac{1}{(2 \pi i)^{2}} \frac{\partial^{2}}{\partial z^{2}},
$$

then

$$
\mathrm{H} \mathcal{R}=2 \eta^{2} \mathcal{C}^{3},
$$

where $\eta$ is the Dedekind $\eta$-function, given by $\eta(\tau)=q^{1 / 24} \prod_{n=1}^{\infty}\left(1-q^{n}\right)$.

Note that the identity found in [2] is slightly different, because they use a different normalization. The two identities, however, are easily seen to be equivalent.

In [4] it is explained how the Rank-Crank PDE fits naturally into the theory of (non-holomorphic) Jacobi forms and a generalization is given for an infinite family of related functions. The method used, however, works only in certain special cases and no results are found for the level $l$ Appell function

$$
A_{l}(z ; \tau):=w^{l / 2} \sum_{n \in \mathbb{Z}} \frac{(-1)^{\ln } q^{\frac{l}{2} n(n+1)}}{1-w q^{n}}, \quad l \in \mathbb{Z}_{>0},
$$

for values of $l$ higher than 3. See [10] for more details on Appell functions and their behaviour under modular transformation properties.

Remark 1.3. Just as $A_{1} / \eta$ is related to cranks, and $A_{3} / \eta$ is related to ranks, $A_{l} / \eta$ for odd $l$ also has an interpretation in terms of partition 
statistics: it is related to the so called $(l+1) / 2$-rank of a partition. See [7] for more details and some background on partition statistics in general.

Somewhat surprisingly, Garvan ([8]) found a PDE for a level 5 Appell function. His original equation involves 10 terms and we will not reproduce it here. However, for a slightly different normalization, his equation simplifies considerably to give

TheOREM 1.4 (Garvan). Let

$$
G_{5}(z ; \tau):=\frac{A_{5}(z ; \tau)}{\eta(\tau)^{3}}
$$

and define the heat operator

$$
\mathrm{H}:=\frac{5}{\pi i} \frac{\partial}{\partial \tau}+\frac{1}{(2 \pi i)^{2}} \frac{\partial^{2}}{\partial z^{2}} .
$$

Then

$$
\left(\mathrm{H}^{2}-E_{4}\right) G_{5}=24 \eta^{2} \mathcal{C}^{5}
$$

where $E_{4}$ is the usual Eisenstein series

$$
E_{4}(\tau)=1+240 \sum_{n=1}^{\infty}\left(\sum_{d \mid n} d^{3}\right) q^{n} .
$$

Garvan's result turns out to be a special case of the following

Theorem 1.5. Let $l \geq 3$ be an odd integer. Define

$$
\begin{aligned}
\mathcal{H}_{k} & :=\frac{l}{\pi i} \frac{\partial}{\partial \tau}+\frac{1}{(2 \pi i)^{2}} \frac{\partial^{2}}{\partial z^{2}}-\frac{l(2 k-1)}{12} E_{2}, \\
\mathcal{H}^{k} & :=\mathcal{H}_{2 k-1} \mathcal{H}_{2 k-3} \cdots \mathcal{H}_{3} \mathcal{H}_{1},
\end{aligned}
$$

where $E_{2}(\tau)=1-24 \sum_{n=1}^{\infty}\left(\sum_{d \mid n} d\right) q^{n}$ is the usual Eisenstein series of weight 2. Then there exist holomorphic modular forms $f_{j}$ on $\mathrm{SL}_{2}(\mathbb{Z})$ of weight $j$, with $j=4,6,8, \ldots, l-1$, such that

$$
\left(\mathcal{H}^{(l-1) / 2}+\sum_{k=0}^{(l-5) / 2} f_{l-2 k-1} \mathcal{H}^{k}\right) A_{l}=(l-1) ! \eta^{l} \mathcal{C}^{l} .
$$

For the proof of the theorem we will need the following

Lemma 1.6. Let $l \geq 3$ be an odd integer. Define

$$
\begin{aligned}
\mathrm{D}_{k} & :=\frac{1}{2 \pi i} \frac{\partial}{\partial \tau}-\frac{k}{12} E_{2}, \\
\mathrm{D}^{k} & :=\mathrm{D}_{2 k-3 / 2} \mathrm{D}_{2 k-7 / 2} \cdots \mathrm{D}_{5 / 2} \mathrm{D}_{1 / 2},
\end{aligned}
$$

and for $r \in \mathbb{Z}$ and $\tau \in \mathbb{H}$,

$$
\vartheta_{l, r}(\tau):=\sum_{n \in \mathbb{Z}}(-1)^{n} q^{\frac{l}{2}(n-1 / 2+r / l)^{2}} .
$$


Then there exist holomorphic modular forms $F_{j}$, with $j=4,6,8, \ldots, l-1$, on $\mathrm{SL}_{2}(\mathbb{Z})$ of weight $j$, such that

$$
\left(\mathrm{D}^{(l-1) / 2}+\sum_{k=0}^{(l-5) / 2} F_{l-2 k-1} \mathrm{D}^{k}\right) \vartheta_{l, r}=0
$$

for all $r \in \mathbb{Z}$.

REMARK 1.7. In the proofs we will see an explicit construction for the $F_{j}$ 's and $f_{j}$ 's for given $l$.

In the next section we will prove Theorem 1.5 and Lemma 1.6, and in Section 3 we will look at the first few cases and in particular we will see that the theorem for $l=5$ is equivalent to Theorem 1.4 .

\section{Proofs of Theorem 1.5 and Lemma 1.6}

Proof of Theorem 1.5. Throughout we assume that $l \geq 3$ is an odd integer. We (trivially) have

$$
A_{l}(z+1 ; \tau)=-A_{l}(z ; \tau),
$$

and if we replace $z$ by $z+\tau$ and $n$ by $n-1$ in 1.2 we find

$$
e^{-2 \pi i l z-\pi i l \tau} A_{l}(z+\tau ; \tau)=-w^{-l / 2} \sum_{n \in \mathbb{Z}} \frac{(-1)^{n} q^{\frac{l}{2} n(n-1)}}{1-w q^{n}},
$$

and so

$$
\begin{aligned}
A_{l}(z ; \tau)+e^{-2 \pi i l z-\pi i l \tau} A_{l}(z+\tau ; \tau) & \\
= & -w^{-l / 2} \sum_{n \in \mathbb{Z}} \frac{(-1)^{n} q^{\frac{l}{2} n(n-1)}}{1-w q^{n}}\left(1-w^{l} q^{l n}\right) \\
& =-w^{-l / 2} \sum_{n \in \mathbb{Z}}(-1)^{n} q^{\frac{l}{2} n(n-1)} \sum_{r=0}^{l-1} w^{r} q^{n r} \\
& =-\sum_{r=0}^{l-1} w^{r-l / 2} q^{-\frac{1}{2 l}(r-l / 2)^{2}} \sum_{n \in \mathbb{Z}}(-1)^{n} q^{\frac{l}{2}(n-1 / 2+r / l)^{2}} \\
& =-\sum_{r=0}^{l-1} e^{2 \pi i(r-l / 2) z-\frac{\pi i}{l}(r-l / 2)^{2} \tau} \vartheta_{l, r}(\tau),
\end{aligned}
$$

with $\vartheta_{l, r}$ as defined in Lemma 1.6 .

It is easy to check that

$$
\left(\frac{l}{\pi i} \frac{\partial}{\partial \tau}+\frac{1}{(2 \pi i)^{2}} \frac{\partial^{2}}{\partial z^{2}}\right) e^{2 \pi i(r-l / 2) z-\frac{\pi i}{l}(r-l / 2)^{2} \tau}=0,
$$


and that for functions $F: \mathbb{C} \times \mathbb{H} \rightarrow \mathbb{C}$,

$$
\begin{aligned}
\mathcal{H}_{k}(F(z+1 ; \tau)) & =\left(\mathcal{H}_{k} F\right)(z+1 ; \tau), \\
\mathcal{H}_{k}\left(e^{-2 \pi i l z-\pi i l \tau} F(z+\tau ; \tau)\right) & =e^{-2 \pi i l z-\pi i l \tau}\left(\mathcal{H}_{k} F\right)(z+\tau ; \tau),
\end{aligned}
$$

with $\mathcal{H}_{k}$ as in the theorem. Hence we get, from applying $\mathcal{H}_{1}$ to equations 2.1) and (2.2),

$$
\left(\mathcal{H}_{1} A_{l}\right)(z+1 ; \tau)=-\left(\mathcal{H}_{1} A_{l}\right)(z ; \tau)
$$

and

$$
\begin{aligned}
\left(\mathcal{H}_{1} A_{l}\right)(z ; \tau)+e^{-2 \pi i l z-\pi i l \tau}\left(\mathcal{H}_{1} A_{l}\right)(z+\tau ; \tau) & \\
= & -2 l \sum_{r=0}^{l-1} e^{2 \pi i(r-l / 2) z-\frac{\pi i}{l}(r-l / 2)^{2} \tau}\left(\mathrm{D}_{1 / 2} \vartheta_{l, r}\right)(\tau),
\end{aligned}
$$

with the operator $\mathrm{D}_{k}$ as defined in Lemma 1.6 .

If we successively apply $\mathcal{H}_{3}, \mathcal{H}_{5}, \ldots$, up to $\mathcal{H}_{2 k-1}$ we find

$$
\left(\mathcal{H}^{k} A_{l}\right)(z+1 ; \tau)=-\left(\mathcal{H}^{k} A_{l}\right)(z ; \tau),
$$

and

$$
\begin{aligned}
\left(\mathcal{H}^{k} A_{l}\right)(z ; \tau)+e^{-2 \pi i l z-\pi i l \tau}\left(\mathcal{H}^{k} A_{l}\right)(z+\tau ; \tau) & \\
= & -(2 l)^{k} \sum_{r=0}^{l-1} e^{2 \pi i(r-l / 2) z-\frac{\pi i}{l}(r-l / 2)^{2} \tau}\left(\mathrm{D}^{k} \vartheta_{l, r}\right)(\tau),
\end{aligned}
$$

with $\mathrm{D}^{k}$ as in Lemma 1.6 .

Define

$$
P:=\left(\mathcal{H}^{(l-1) / 2}+\sum_{k=0}^{(l-5) / 2} f_{l-2 k-1} \mathcal{H}^{k}\right) A_{l},
$$

with

$$
f_{j}=(2 l)^{j / 2} F_{j}, \quad j=4,6, \ldots, l-1,
$$

and $F_{j}$ as in the lemma. Then we see from equations $(2.3)$ and $(2.4)$ that

$$
P(z+1 ; \tau)=e^{-2 \pi i l z-\pi i l \tau} P(z+\tau ; \tau)=-P(z ; \tau) .
$$

Now consider the Jacobi theta function

$$
\begin{aligned}
\vartheta(z ; \tau) & :=\sum_{n \in \mathbb{Z}}(-1)^{n} w^{n+1 / 2} q^{\frac{1}{2}(n+1 / 2)^{2}} \\
& =w^{1 / 2} q^{1 / 8} \prod_{n=1}^{\infty}\left(1-q^{n}\right)\left(1-w q^{n}\right)\left(1-w^{-1} q^{n-1}\right)=-\frac{\eta(\tau)^{2}}{\mathcal{C}(z ; \tau)} .
\end{aligned}
$$

This function satisfies

$$
\vartheta(z+1 ; \tau)=e^{2 \pi i z+\pi i \tau} \vartheta(z+\tau ; \tau)=-\vartheta(z ; \tau),
$$


$z \mapsto \vartheta(z ; \tau)$ has simple zeros in $\mathbb{Z} \tau+\mathbb{Z}$ and

$$
\left.\frac{1}{2 \pi i} \frac{\partial}{\partial z}\right|_{z=0} \vartheta(z ; \tau)=\eta(\tau)^{3} .
$$

Since the poles of $z \mapsto A_{l}(z ; \tau)$ are simple poles in $\mathbb{Z} \tau+\mathbb{Z}$, the function $z \mapsto P(z ; \tau)$ has poles of order $l$ in $\mathbb{Z} \tau+\mathbb{Z}$, and so the function

$$
p(z ; \tau):=\vartheta(z ; \tau)^{l} P(z ; \tau)
$$

is holomorphic as a function of $z$. Using (2.5) and (2.6) we find that

$$
p(z+1 ; \tau)=p(z+\tau ; \tau)=p(z ; \tau) .
$$

We see that $z \mapsto p(z ; \tau)$ is a double periodic holomorphic function on $\mathbb{C}$ and hence by Liouville's Theorem, constant. To determine the constant, we consider the behaviour for $z \rightarrow 0$. From (1.2) we find that for $z \rightarrow 0$,

$$
A_{l}(z ; \tau)=-\frac{1}{2 \pi i} \frac{1}{z}+\mathcal{O}(1)
$$

and so

$$
P(z ; \tau)=-\frac{(l-1) !}{(2 \pi i)^{l}} \frac{1}{z^{l}}+\mathcal{O}\left(\frac{1}{z^{l-1}}\right) .
$$

Combining this with 2.7 we see that

$$
p(z ; \tau)=-(l-1) ! \eta(\tau)^{3 l},
$$

and so

$$
P(z ; \tau)=-(l-1) ! \frac{\eta(\tau)^{3 l}}{\vartheta(z ; \tau)^{l}}=(l-1) ! \eta(\tau)^{l} \mathcal{C}(z ; \tau)^{l},
$$

which finishes the proof.

Proof of Lemma 1.6. Throughout, let $l \geq 3$ be an odd integer. Because of the trivial relations

$$
\vartheta_{l, r+l}=-\vartheta_{l, r}, \quad \vartheta_{l,-r}=-\vartheta_{l, r},
$$

it suffices to consider $\vartheta_{l, r}$ for $r=1,2, \ldots,(l-1) / 2$. Define

$$
\Theta_{l}:=\left(\begin{array}{c}
\vartheta_{l, 1} \\
\vartheta_{l, 2} \\
\vdots \\
\vartheta_{l,(l-1) / 2}
\end{array}\right) .
$$

Then $\Theta_{l}$ transforms as a (vector-valued) modular form of weight $1 / 2$ on the full modular group $\mathrm{SL}_{2}(\mathbb{Z})$ :

$$
\begin{aligned}
& \Theta_{l}(\tau+1)=\operatorname{diag}\left(\zeta_{8 l}^{(l-2 j)^{2}}\right)_{1 \leq j \leq(l-1) / 2} \Theta_{l}(\tau), \\
& \Theta_{l}(-1 / \tau)=(-1)^{(l+1) / 2} \sqrt{\tau / l i}(2 \sin 2 \pi r k / l)_{1 \leq r, k \leq(l-1) / 2} \Theta_{l}(\tau) .
\end{aligned}
$$


The first equation is trivial and the second can be obtained by using Poisson summation. We leave the details to the reader. Note that for the proof of the lemma, we actually do not need to know the explicit transformation properties. It would suffice to know that $\Theta_{l}$ is a modular form of weight $1 / 2$ on the full modular group.

Using

$$
E_{2}\left(\frac{a \tau+b}{c \tau+d}\right)=(c \tau+d)^{2} E_{2}(\tau)+\frac{6}{\pi i} c(c \tau+d) \quad \text { for }\left(\begin{array}{ll}
a & b \\
c & d
\end{array}\right) \in \mathrm{SL}_{2}(\mathbb{Z})
$$

(see [9, p. 19]) we can easily verify that

$$
\mathrm{D}_{k}\left((c \tau+d)^{-k} f\left(\frac{a \tau+b}{c \tau+d}\right)\right)=(c \tau+d)^{-k-2}\left(\mathrm{D}_{k} f\right)\left(\frac{a \tau+b}{c \tau+d}\right),
$$

and so

$$
\mathrm{D}^{k}\left((c \tau+d)^{-1 / 2} \Theta_{l}\left(\frac{a \tau+b}{c \tau+d}\right)\right)=(c \tau+d)^{-2 k-1 / 2}\left(\mathrm{D}^{k} \Theta_{l}\right)\left(\frac{a \tau+b}{c \tau+d}\right) .
$$

Now define the $(l-1) / 2 \times(l-1) / 2$-matrix

$$
T_{l}:=\left(\begin{array}{lllll}
\Theta_{l} & \mathrm{D}^{1} \Theta_{l} & \mathrm{D}^{2} \Theta_{l} & \cdots & \mathrm{D}^{(l-3) / 2} \Theta_{l}
\end{array}\right) .
$$

Then $T_{l}$ transforms as a (matrix-valued) modular form on the full modular group $\mathrm{SL}_{2}(\mathbb{Z})$ :

$$
\begin{aligned}
T_{l}(\tau+1)= & \operatorname{diag}\left(\zeta_{8 l}^{(l-2 j)^{2}}\right)_{1 \leq j \leq(l-1) / 2} T_{l}(\tau), \\
T_{l}(-1 / \tau)= & (-1)^{(l+1) / 2} \sqrt{\tau / l i}(2 \sin 2 \pi r k / l)_{1 \leq r, k \leq(l-1) / 2} \\
& \cdot T_{l}(\tau) \operatorname{diag}\left(\tau^{2 j-2}\right)_{1 \leq j \leq(l-1) / 2} .
\end{aligned}
$$

From this we see that

$$
\begin{aligned}
& \operatorname{det}\left(T_{l}(\tau+1)\right)=\zeta_{24}^{(l-1)(l-2) / 2} \operatorname{det}\left(T_{l}(\tau)\right), \\
& \operatorname{det}\left(T_{l}(-1 / \tau)\right)=(-i \tau)^{(l-1)(l-2) / 4} \operatorname{det}\left(T_{l}(\tau)\right),
\end{aligned}
$$

and so $\operatorname{det}\left(T_{l}\right)$ is a multiple of $\eta^{(l-1)(l-2) / 2}$. We determine what that multiple is by looking at the lowest order terms:

First observe that by doing elementary column operations we get

$$
\operatorname{det}\left(T_{l}\right)=\operatorname{det}\left(\begin{array}{lllll}
\Theta_{l} & \partial_{\tau} \Theta_{l} & \partial_{\tau}^{2} \Theta_{l} & \cdots & \partial_{\tau}^{(l-3) / 2} \Theta_{l}
\end{array}\right),
$$

with $\partial_{\tau}:=\frac{1}{2 \pi i} \frac{\partial}{\partial \tau}$.

For $1 \leq r \leq(l-1) / 2$ we have

$$
\vartheta_{l, r}(\tau)=q^{(l-2 r)^{2} / 8 l}(1+\mathcal{O}(q))
$$


so

$\left(\begin{array}{lllll}\Theta_{l} & \partial_{\tau} \Theta_{l} & \partial_{\tau}^{2} \Theta_{l} & \cdots & \partial_{\tau}^{(l-3) / 2} \Theta_{l}\end{array}\right)$

$=\operatorname{diag}\left(q^{(l-2 i)^{2} / 8 l}\right)_{1 \leq i \leq(l-1) / 2} \cdot\left(\left(\frac{(l-2 i)^{2}}{8 l}\right)^{j-1}+\mathcal{O}(q)\right)_{1 \leq i, j \leq(l-1) / 2}$,

$\operatorname{det}\left(\Theta_{l} \quad \partial_{\tau} \Theta_{l} \quad \partial_{\tau}^{2} \Theta_{l} \quad \cdots \quad \partial_{\tau}^{(l-3) / 2} \Theta_{l}\right)=q^{(l-1)(l-2) / 48}(\operatorname{det}(B)+\mathcal{O}(q))$,

and hence

$$
\operatorname{det}\left(T_{l}(\tau)\right)=\operatorname{det}(B) \eta(\tau)^{(l-1)(l-2) / 2}
$$

with

$$
B_{i j}=\left(\frac{(l-2 i)^{2}}{8 l}\right)^{j-1} \quad \text { for } 1 \leq i, j \leq(l-1) / 2 .
$$

$B$ is a Vandermonde matrix: an $m \times n$ matrix $V$ such that $V_{i j}=\alpha_{i}^{j-1}$ with $\alpha_{i} \in \mathbb{R}$. Since a square Vandermonde matrix is invertible if and only if the $\alpha_{i}$ are distinct, we see that $B$ is invertible. From (2.9) and the fact that $\eta$ has no zeros on $\mathbb{H}$ we then conclude that $T_{l}(\tau)$ is invertible for all $\tau \in \mathbb{H}$.

Now define the $(l-1) / 2$ functions $F_{j}$ with $j=2,4, \ldots, l-1$ by

$$
\left(\begin{array}{c}
F_{l-1} \\
F_{l-3} \\
\vdots \\
F_{2}
\end{array}\right):=-T_{l}^{-1} \mathrm{D}^{(l-1) / 2} \Theta_{l} .
$$

We claim that the $F_{j}$ are holomorphic modular forms of weight $j$ on $\mathrm{SL}_{2}(\mathbb{Z})$. The modular transformation properties follow easily from (2.8) and those of $\mathrm{D}^{(l-1) / 2} \Theta_{l}$, which are

$$
\begin{aligned}
& \mathrm{D}^{(l-1) / 2} \Theta_{l}(\tau+1)=\operatorname{diag}\left(\zeta_{8 l}^{(l-2 j)^{2}}\right)_{1 \leq j \leq(l-1) / 2} \mathrm{D}^{(l-1) / 2} \Theta_{l}(\tau) \\
& \mathrm{D}^{(l-1) / 2} \Theta_{l}(-1 / \tau) \\
& \quad=(-1)^{(l+1) / 2} \sqrt{\tau / l i} \tau^{l-1}(2 \sin 2 \pi r k / l)_{1 \leq r, k \leq(l-1) / 2} \mathrm{D}^{(l-1) / 2} \Theta_{l}(\tau) .
\end{aligned}
$$

Since $\operatorname{det}\left(T_{l}\right)$ has no zeros on $\mathbb{H}$ we see that $F_{j}$ is a holomorphic function on $\mathbb{H}$. That it also has no pole at infinity follows from

$$
\begin{aligned}
\mathrm{D}^{(l-1) / 2} \Theta_{l} & =\operatorname{diag}\left(q^{(l-2 i)^{2} / 8 l}\right)_{1 \leq i \leq(l-1) / 2} \cdot\left(\begin{array}{c}
\mathcal{O}(1) \\
\mathcal{O}(1) \\
\vdots \\
\mathcal{O}(1)
\end{array}\right) \\
T_{l} & =\operatorname{diag}\left(q^{(l-2 i)^{2} / 8 l}\right)_{1 \leq i \leq(l-1) / 2} \cdot\left(C_{i j}+\mathcal{O}(q)\right)_{1 \leq i, j \leq(l-1) / 2}
\end{aligned}
$$


for some $(l-1) / 2 \times(l-1) / 2$-matrix $C$, with

$$
\operatorname{det}(C)=\operatorname{det}(B) \neq 0 .
$$

Since there are no holomorphic modular forms of weight 2 on $\mathrm{SL}_{2}(\mathbb{Z})$, we have $F_{2} \equiv 0$, and so we can rewrite 2.10 as

$$
\mathrm{D}^{(l-1) / 2} \Theta_{l}+T_{l}\left(\begin{array}{c}
F_{l-1} \\
\vdots \\
F_{4} \\
0
\end{array}\right)=0,
$$

which is equivalent to the condition that 1.3 holds for $1 \leq r \leq(l-1) / 2$.

3. Some examples. For $l=3$, the sum $\sum_{k=0}^{(l-5) / 2}$ is empty, and so Theorem 1.5 gives

$$
\mathcal{H}_{1} A_{3}=2 \eta^{3} \mathcal{C}^{3} \text {. }
$$

Using

we see

$$
\mathcal{R}(z ; \tau)=\frac{A_{3}(z ; \tau)}{\eta(\tau)}+e^{\pi i z-\pi i \tau / 12}
$$

$$
\mathcal{H}_{1 / 2} \mathcal{R}=\mathcal{H}_{1 / 2}\left(\frac{A_{3}}{\eta}\right)=\frac{\mathcal{H}_{1} A_{3}}{\eta}+6 A_{3} \mathrm{D}_{-1 / 2}\left(\frac{1}{\eta}\right)=2 \eta^{2} \mathcal{C}^{3}
$$

which is the Rank-Crank PDE. Here we used

$$
\mathrm{D}_{k / 2}\left(\eta^{k}\right)=0
$$

which follows from

$$
E_{2}=24 \frac{\partial_{\tau} \eta}{\eta}
$$

(first equation in the proof of Proposition 7 of [9] together with $\Delta=\eta^{24}$ ).

For $l=5$, we find from 2.10 that

$$
F_{4}(\tau)=-\frac{11}{3600}-\frac{11}{15} q+\mathcal{O}\left(q^{2}\right)
$$

and since $F_{4}$ is a holomorphic modular form on $\mathrm{SL}_{2}(\mathbb{Z})$, we can easily identify it as

and so

$$
F_{4}=-\frac{11}{3600} E_{4}
$$

$$
f_{4}=-\frac{11}{36} E_{4}
$$

If we put this into Theorem 1.5 we get

$$
\left(\mathcal{H}_{3} \mathcal{H}_{1}-\frac{11}{36} E_{4}\right) A_{5}=24 \eta^{5} \mathcal{C}^{5}
$$


We now rewrite this in terms of $G_{5}$ :

$$
\begin{aligned}
\mathcal{H}_{1} A_{5} & =\mathcal{H}_{1}\left(\eta^{3} G_{5}\right)=10\left(\mathrm{D}_{3 / 2} \eta^{3}\right) G_{5}+\eta^{3} \mathcal{H}_{-1 / 2} G_{5}=\eta^{3} \mathcal{H}_{-1 / 2} G_{5}, \\
\mathcal{H}_{3} \mathcal{H}_{1} A_{5} & =\mathcal{H}_{3}\left(\eta^{3} \mathcal{H}_{-1 / 2} G_{5}\right) \\
& =10\left(\mathrm{D}_{3 / 2} \eta^{3}\right) \mathcal{H}_{-1 / 2} G_{5}+\eta^{3} \mathcal{H}_{3 / 2} \mathcal{H}_{-1 / 2} G_{5}=\eta^{3} \mathcal{H}_{3 / 2} \mathcal{H}_{-1 / 2} G_{5},
\end{aligned}
$$

and so we get

$$
\left(\mathcal{H}_{3 / 2} \mathcal{H}_{-1 / 2}-\frac{11}{36} E_{4}\right) G_{5}=24 \eta^{2} \mathcal{C}^{5}
$$

Using

$$
\mathcal{H}_{3 / 2} \mathcal{H}_{-1 / 2}=\mathrm{H}^{2}+\frac{25}{3}\left(\partial_{\tau} E_{2}-\frac{1}{12} E_{2}^{2}\right)
$$

and

$$
\partial_{\tau} E_{2}=\frac{E_{2}^{2}-E_{4}}{12}
$$

(see Proposition 15 of [9]), we see that is equivalent to the statement of Theorem 1.4.

For $l=7$ we find, again using 2.10,

$$
f_{4}=-\frac{35}{9} E_{4} \quad \text { and } \quad f_{6}=\frac{85}{27} E_{6} .
$$

For $l=9$,

$$
f_{4}=-\frac{39}{2} E_{4}, \quad f_{6}=53 E_{6}, \quad f_{8}=-\frac{759}{16} E_{8} .
$$

For $l=11$,

$$
f_{4}=-\frac{583}{9} E_{4}, \quad f_{6}=\frac{3245}{9} E_{6}, \quad f_{8}=-\frac{67661}{81} E_{8}, \quad f_{10}=\frac{252416}{243} E_{10} .
$$

And for $l=13$,

$$
\begin{gathered}
f_{4}=-\frac{6097}{36} E_{4}, \quad f_{6}=\frac{42653}{27} E_{6}, \quad f_{8}=-\frac{8081645}{1296} E_{8}, \\
f_{10}=\frac{4910555}{324} E_{10}, \quad f_{12}=-\frac{1462986875}{46656} E_{12}+\frac{57480372950}{6219} \Delta .
\end{gathered}
$$

In fact, for general $l$ we can show

$$
f_{4}=-\frac{l(l+1)(l-1)(l-3)}{8640}(7 l-24) E_{4},
$$

and further

$$
f_{6}=\frac{l(l+1)(l-1)(l-3)(l-5)}{362880}\left(33 l^{2}-184 l+96\right) E_{6},
$$




$$
\begin{aligned}
f_{8}= & \frac{l(l+1)(l-1)(l-3)(l-5)(l-7)}{1045094400} \\
& \cdot\left(343 l^{4}-12765 l^{3}+75600 l^{2}-60480 l+41472\right) E_{8}, \\
f_{10}= & -\frac{l(l+1)(l-1)(l-3)(l-5)(l-7)(l-9)}{34488115200} \\
& \cdot\left(2541 l^{5}-64399 l^{4}+370624 l^{3}-279264 l^{2}+338688 l-276480\right) E_{10},
\end{aligned}
$$

etc.

Acknowledgements. The author wishes to thank Frank Garvan and Karl Mahlburg for helpful discussions and Kathrin Bringmann for her comments on the first version.

\section{References}

[1] G. E. Andrews and F. G. Garvan, Dyson's crank of a partition, Bull. Amer. Math. Soc. (N.S.) 18 (1988), 167-171.

[2] A. O. L. Atkin and F. G. Garvan, Relations between the ranks and cranks of partitions, Ramanujan J. 7 (2003), 343-366.

[3] A. O. L. Atkin and H. P. F. Swinnerton-Dyer, Some properties of partitions, Proc. London Math. Soc. 4 (1954), 84-106.

[4] K. Bringmann and S. P. Zwegers, Rank-crank type PDE's and non-holomorphic Jacobi forms, Math. Res. Lett., to appear.

[5] F. Dyson, Some guesses in the theory of partitions, Eureka (Cambridge) 8 (1944), $10-15$.

[6] M. Eichler and D. B. Zagier, The Theory of Jacobi Forms, Progr. Math. 55, Birkhäuser, 1985.

[7] F. G. Garvan, Generalizations of Dyson's rank and non-Rogers-Ramanujan partitions, Manuscripta Math. 84 (1994), 343-359.

[8] - , personal communication.

[9] D. B. Zagier, Elliptic modular forms and their applications, in: The 1-2-3 of Modular Forms, Universitext, Springer, Berlin, 2008, 1-103.

[10] S. P. Zwegers, Mock theta functions I: Appell functions and the Mordell integral, in preparation.

Sander Zwegers

School of Mathematical Sciences

University College Dublin

Belfield, Dublin 4, Ireland

E-mail: sander.zwegers@ucd.ie

Received on 8.9.2009

and in revised form on 1.2.2010 